\title{
COMMUTATORS OF POTENTIAL TYPE OPERATORS WITH LIPSCHITZ SYMBOLS ON VARIABLE LEBESGUE SPACES WITH DIFFERENT WEIGHTS
}

\section{Luciana Melchiori, Gladis Pradolini And Wilfredo Ramos}

Abstract. We prove that a generalized Fefferman-Phong type condition on a pair of weights $u$ and $v$ is sufficient for the boundedness of the commutators of potential type operators from $L_{v}^{p(\cdot)}$ into $L_{u}^{q(\cdot)}$. We also give an improvement of this result in the sense that we not only consider a variable version of power bump conditions, but also weaker norms related to Musielak-Orlicz functions.

We consider a wider class of symbols including Lipschitz symbols and some generalizations.

Mathematics subject classification (2010): 42B25.

Keywords and phrases: Potential operators, commutators, variable Lebesgue spaces.

\section{REFERENCES}

[1] A. Bernardis, O. Gorosito And G. Pradolini, Weighted inequalities for multilinear potential operators and their commutators, Potential Anal.35, 3, 2011, 253-274.

[2] E. Dalmasso, G. Pradolini And W. Ramos, The effect of the smoothness of fractional type operators over their commutators with Lipschitz symbols on weighted spaces, Fract. Calc. Appl. Anal. $21,3,2018,628-653$.

[3] L. Diening, Maximal function on Musielak-Orlicz spaces and generalized Lebesgue spaces, Bull. Sci. Math. 129, 8, 2005, 657-700.

[4] L. Diening, P. Harjulehto, P. HÄStÖ, And M. RuZicka, Lebesgue and Sobolev spaces with variable exponents, Lecture Notes in Mathematics, Springer 2017, Heidelberg, 2011.

[5] P. Harjulehto And P. HÄstö, Orlicz Spaces and Generalized Orlicz Spaces, Preprint http://cc.oulu.fi/ phasto/pp/orliczBook.pdf., 2018.

[6] W. LI, John-Nirenberg inequality and self-improving properties, J. Math. Res. Exposition 25, 1, 2005, $42-46$.

[7] W. LI, Two-weight norm inequalities for commutators of potential type integral operators, J. Math. Anal. Appl. 322, 2, 2006, 1215-1223.

[8] W. LI, Weighted inequalities for commutators of potential type operators, J. Korean Math. Soc. 44, 6, 2007, 1233-1241.

[9] W. LI, J. Y. QI AND X. F. YAN, Weighted norm inequalities for potential type operators, J. Math. Res. Exposition 29, 5, 2009, 895-900.

[10] F. Maeda, Y. Mizuta And T. Ohno, Approximate identities and Young type inequalities in variable Lebesgue-Orlicz spaces $L^{p(\cdot)}(\log L)^{q(\cdot)}$, Ann. Acad. Sci. Fenn. Math. 35, 2, 2010, 405-420.

[11] L. Melchiori and G. Pradolini, Potential operators and their commutators acting between variable Lebesgue spaces with different weights, Integral Transforms Spec. Funct. 29, 11, 2018, 909-926.

[12] Y. Meng AND D. YANG, Boundedness of commutators with Lipschitz functions in non-homogeneous spaces, Taiwanese J. Math. 10, 6, 2006, 1443-1464.

[13] B. Muckenhoupt AND R. Wheeden, Weighted norm inequalities for fractional integrals, Trans. Amer. Math. Soc. 192, 1, 1974, 261-274. 
[14] C. PÉREZ, Two weighted inequalities for potential and fractional type maximal operators, Indiana Univ. Math. J. 43, 2, 1994, 663-683.

[15] G. Pradolini AND W. Ramos, Characterization of Lipschitz functions via the commutators of singular and fractional integral operators in variable Lebesgue spaces, Potential Anal. 46, 3, 2017, 499-525.

[16] M. M. Rao And Z. D. Ren, Theory of Orlicz spaces 146, Monographs and Textbooks in Pure and Applied Mathematics, Marcel Dekker, Inc., New York, 1991.

[17] E. SAWYER AND R. L. WheEDEN, Weighted inequalities for fractional integrals on Euclidean and homogeneous spaces, Amer. J. Math. 114, 4, 1992, 813-874. 